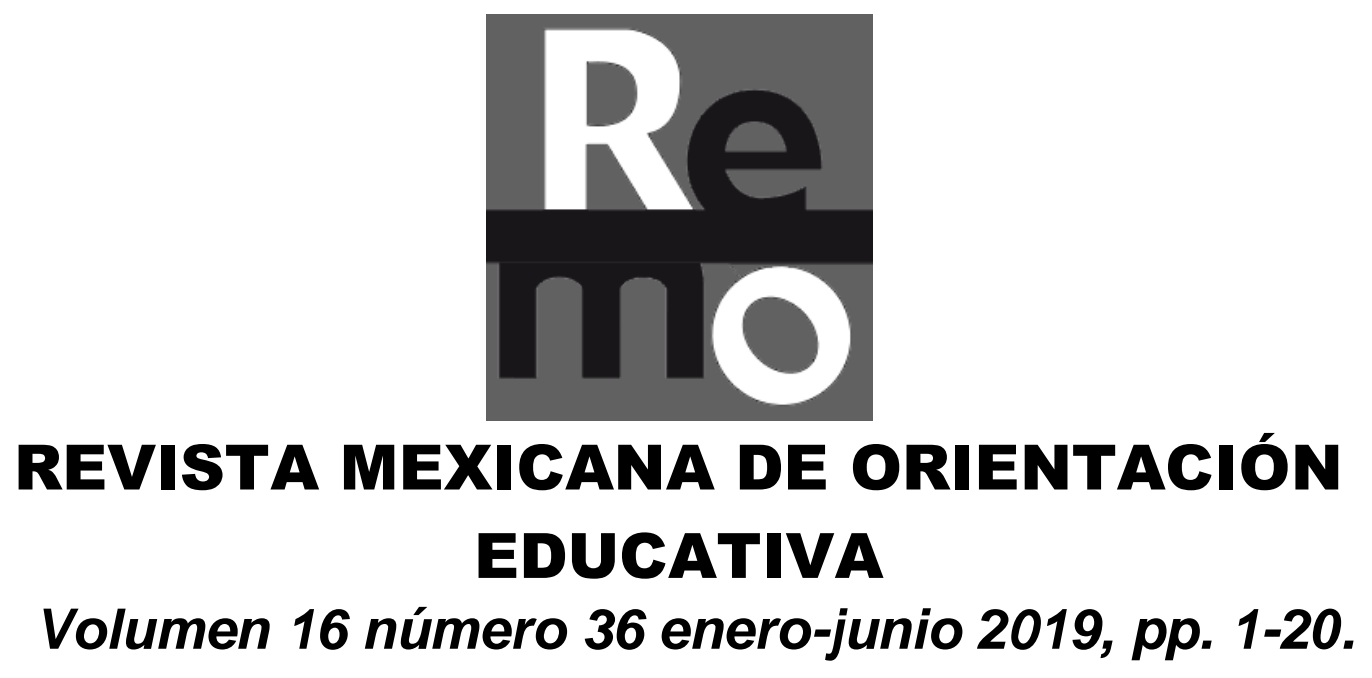

\title{
La interacción en el aula. Un elemento que configura las prácticas de evaluación en la escuela primaria
}

Catalina Cruz Albino*1

Fecha de recepción: 10 de marzo de 2018

Fecha de aprobación 15 de enero de 2019

DOI: https://doi.org/10.31206/rmdo122019

\begin{abstract}
RESUMEN
El presente artículo se deriva de la investigación "Prácticas de evaluación en el aula de cinco docentes en dos escuelas primarias en el municipio de Toluca" el cual se desarrolla mediante los principios fundamentales del paradigma cualitativo con enfoque metodológico de la etnografía educativa para documentar las prácticas de evaluación que los docentes realizan en el aula al identificar los principales elementos que configuran dichas prácticas. Se parte
\end{abstract}

\footnotetext{
1 *Doctorante en el Instituto Superior de Ciencias de la Educación del Estado de México. ISCEEM. Toluca, Edo. de México. Tel. 7225832553. katyprof@ hotmail.com.
} 
del supuesto de que las prácticas de evaluación en el aula de algunos docentes de educación primaria se enfocan en traducir a la evaluación en una calificación para cumplir con la demanda institucional y no con la finalidad de evaluar para mejorar, esto con el objetivo de describir, interpretar y comprender las prácticas de evaluación en el aula mediante el análisis de los elementos y rasgos característicos del contexto y los distintos referentes de la teoría.

Palabras clave: Escuela primaria, interacción, práctica docente, prácticas de evaluación en el aula.

\begin{abstract}
This article is derived from the research "Classroom assessment practices of five teachers in two primary schools in the municipality of Toluca" which is developed through the fundamental principles of the qualitative paradigm with a methodological approach to educational ethnography to document the practices of evaluation that teachers perform in the classroom by identifying the main elements that make up these practices. It is assumed that the evaluation practices in the classroom of some primary education teachers focus on translating the evaluation into a qualification to meet institutional demand and not with the purpose of evaluating to improve, this with the objective of describe, interpret and understand the evaluation practices in the classroom through the analysis of the elements and characteristic features of the context and the different referents of the theory.
\end{abstract}

Keywords: Primary school, classroom evaluation, teaching practice, assessment practices, interaction.

\title{
RESUMO
}

Este artigo é derivado da pesquisa "Práticas de avaliação em sala de aula de cinco professores em duas escolas primárias do município de Toluca" que é desenvolvida através dos princípios fundamentais do paradigma qualitativo com uma abordagem metodológica à etnografia educacional para documentar as práticas de avaliação que os professores realizam em sala de aula identificando os principais elementos que compõem essas práticas. Pressupõe-se que as práticas de avaliação em sala de aula de alguns professores de educação primária se concentram em traduzir a avaliação em uma qualificação para atender a demanda institucional e não com o propósito de avaliar para melhorar, isto com o objetivo de descrever, interpretar e compreender as práticas de avaliação em sala de aula através da análise dos elementos e características do contexto e os diferentes referentes da teoria. 
Palavras-chave: Ensino fundamental, interação, prática de ensino, práticas de avaliação em sala de aula.

\section{PRESENTACIÓN}

El presente artículo forma parte de una investigación que se desarrolla en el marco del programa doctoral en "Ciencias de la Educación" del Instituto Superior de Ciencias de la Educación del Estado de México (ISCEEM). En él se exponen algunas de las prácticas de evaluación que desarrollan los docentes en la escuela primaria, estas prácticas dan cuenta de los rasgos que configura cada docente así como el contexto del aula y las necesidades académicas que allí se presentan.

Como fenómeno social, la evaluación en el aula es una práctica fundamental en la función docente, la manera en conceptualizarla y desarrollarla en el aula impacta de forma importante en el ámbito escolar. Por lo que en este artículo se dan a conocer algunos antecedentes que dieron origen al objeto de estudio desde la lógica metodológica que plantea Bourdieu, de manera que dicho objeto de estudio se fue construyendo a partir de pre-nociones ${ }^{2}$ como producto de la experiencia a lo largo de 15 años de trabajo con distintos grupos de alumnos. Posteriormente mediante el acercamiento al aula, a los docentes y alumnos dio apertura romper con algunos prejuicios que se tenían, lo que para Bourdieu (2002) son actos de ruptura $^{3}$ para llegar a la reconstrucción y la relación de los hechos desde distintos referentes mismos que encaminaron a la integración de las unidades de análisis de la investigación. Es así que la investigación se desarrolla desde el enfoque cualitativo como paradigma de investigación dado a que "posee un fundamento decididamente humanista para entender la realidad social...percibe la vida social como la creatividad compartida de los individuos" (Reichardt y Cook, 2005: 62), por su carácter interpretativo comprende situaciones desde la perspectiva de los participantes en cada situación, con esto resulta indispensable describir, comprender e interpretar el fenómeno de las prácticas de evaluación tomando en cuenta saberes, modelos de formación, experiencias, opiniones, acciones y todas las condiciones a partir del cual hacen lo que hacen. Es así que por medio de un conjunto de técnicas como la entrevista, la historia de vida y la observación participante se tuvo acercamiento a los

\footnotetext{
${ }^{2}$ Representaciones esquemáticas y sumarias que se forman en la práctica y para ella" (Bourdieu, 2002, p.28).

${ }^{3}$ Sustituir las nociones del sentido común por una primera noción científica (Bourdieu, 2002, p.28).
} 
docentes como sujetos reales que llevan consigo experiencias, saberes y opiniones propias acerca de sus prácticas de evaluación en el aula de clase.

Según la naturaleza del fenómeno educativo, el objeto de estudio, los participantes y el contexto mismo, la investigación se desarrolló desde el enfoque metodológico de la etnografía educativa, el cual permitió mirar lo que las personas hacen habitualmente tal como coinciden Rockwell y Bertely para identificar las recurrencias de los hechos en la vida cotidiana del aula.

Los objetivos planteados en la investigación viabilizaron una relación dialéctica de los conceptos y el trabajo de campo, de manera que al poner en balance los alcances de la investigación se abre una brecha a la reflexión crítica de las prácticas de evaluación en el aula no sólo gracias al aporte de los autores sino a la voz de los participantes de la investigación. El artículo se organiza en cinco apartados: el primero de ellos refiere a los antecedentes del objeto de estudio en el cual se toma en cuenta las nociones previas basadas en la experiencia, así como los principales estudios que se han llevado a cabo sobre prácticas de evaluación en el aula, en el segundo apartado hace un recorrido histórico de la evaluación desde una postura pedagógica, en el tercer apartado se explicitan los rasgos de las prácticas en la escuela primaria que llevan a cabo los docentes el cual amalgama creencias, lenguajes y valores a partir de la formación, en el penúltimo apartado se aborda el trabajo empírico en el que se identifican los rasgos de la interacción en el aula como uno de los elementos que configuran las prácticas de evaluación y finalmente se exponen algunas conclusiones que responden a la realidad que se presenta con base a los límites temporales y espaciales de un contexto y a los avances que hasta el momento se tienen de la investigación.

\section{Construcción del objeto de estudio}

La construcción del objeto de estudio se orientó desde las prácticas de evaluación que hacen los docentes en el aula lo cual implicó tomar en cuenta la realidad inmediata y no quedarse únicamente con las nociones previas, así la reconstrucción de conceptos dio nuevo significado a lo que se vive cotidianamente, en esta lógica no se invalidó el sentido común sino se trató de “...poner en duda la idea de un saber absoluto” (Duhalde, 1999: 45) mismo que tuvo lugar en un proceso de interacción entre distintas disciplinas con la realidad observada de los significados que otorgan los participantes. 


\section{Antecedentes}

\section{DESARROLLO}

A lo largo de 15 años de servicio frente a grupo pude observar que muchos docentes dan mayor importancia a la calificación como fin último de la evaluación, por lo que los reportes cualitativos en los informes de evaluación son muy escasos en relación a los reportes cuantitativos de manera que, también permea la devolución numérica más que una devolución descriptiva de parte del docente. Según referentes de la misma experiencia, en muchas ocasiones durante la práctica de los docentes se tiende a la improvisación y simulación de una evaluación que abandera un discurso de la política educativa sin omitir que para algunos docentes la evaluación representa un requisito administrativo que hay que cumplir en tiempo y forma más que una posibilidad para mejorar el aprendizaje como lo establece el plan de estudios (2011), pues se mira como una exigencia para la rendición de cuentas de manera periódica y no como un proceso reflexivo para mejorar o adecuar las estrategias de enseñanza.

Es así que, la intención y el concepto que los actores educativos tienen de la evaluación toma distintas visiones, es decir; para los alumnos la evaluación es sinónimo de aprobar o reprobar y no precisamente para aprender, para muchos maestros la evaluación es un requisito administrativo que fatiga y estresa, más aún cuando hay que entregar la estadística de un día para otro pues calificar un determinado número de exámenes, sumar de escalas, revisar trabajos individuales y de equipo implica dejar de lado lo importante para atender lo urgente, de tal manera que el análisis de lo que ocurre en el proceso de evaluación en el aula se queda en último término. Por su parte, para los padres de familia la evaluación representa los conocimientos adquiridos en una escala de 5 a 10 que se traduce coloquialmente en la garantía de que los gastos generados para la educación de los hijos están siendo o no aprovechados, sin embargo para algunas autoridades educativas la evaluación se concreta en la rendición de cuentas de que se está avanzando hacia el cumplimiento de los estándares curriculares establecidos aunque esto traiga como consecuencia solamente la acumulación de datos estadísticos generalmente sin traducir.

De manera que, hablar de evaluación en el aula se torna familiar no sólo para el docente sino también para toda persona que ha pasado por un aula de clases pues por mucho tiempo la evaluación se ha visto como una actividad necesaria en la práctica diaria de la formación académica, sin embargo; el tema de la evaluación en el aula parece ser una de las más grandes 
preocupaciones no sólo de las autoridades educativas y de padres de familia sino también de profesores y alumnos. Para muchos alumnos la evaluación es un tema que causa estrés lo que conlleva al desinterés por participar en las distintas actividades que tienen relación con ésta, mientras que la urgente entrega de reportes estadísticos, el empleo privilegiado de exámenes de muchos profesores minimiza la reflexión sobre la importancia y trascendencia de la evaluación.

\section{Algunas investigaciones que destacan las prácticas de evaluación en el aula}

Enseguida retomo algunas investigaciones que han considerado aspectos de las prácticas de evaluación en el aula y que han tenido trascendencia en la investigación. En este sentido Velasco (2006) desarrolla una investigación que titula "Las prácticas cotidianas y los saberes docentes sobre la evaluación de los aprendizajes en la escuela primaria. Un estudio de caso" cuyo propósito consiste en describir y analizar las prácticas y saberes docentes sobre la evaluación de los aprendizajes desarrollados por una profesora de educación primaria en la interacción cotidiana con sus alumnos. Se recurre al estudio de caso para estudiar a la profesora como sujeto histórico, social y discursivo, se enfoca en los estudios realizados por Agnes Heller para el saber docente cotidiano. En este sentido, aborda a la investigación desde la perspectiva etnográfica y la investigación cualitativa, así mismo se rescatan las estrategias (revisión de ejercicios, asignación de calificaciones, valoración de desempeños) y los instrumentos (examen y escala estimativa) utilizadas por la maestra para evaluar los aprendizajes.

Las conclusiones de dicha investigación ponen de manifiesto que las prácticas y saberes que tienen los docentes adquieren significado en la vida cotidiana escolar, argumenta la autora que la práctica docente es compleja porque no se limita al abordaje de contenidos curriculares, sino que en ella intervienen una serie de elementos sociales, culturales y políticos que la mediatizan y la determinan, por lo que, las actividades realizadas en la interacción cotidiana con los alumnos dan muestra de la diversidad de prácticas y saberes

docentes. Finalmente, la investigadora concluye con dos planteamientos para dar pauta a nuevas investigaciones, estas son: ¿Cómo transforman los docentes los saberes teóricos en saberes práctico-teóricos para la enseñanza, el aprendizaje, la evaluación, el uso de recursos materiales de la escuela, la distribución de los tiempos y los espacios escolares, el trabajo con 
los contenidos escolares? y ¿Qué condiciones de la Institución escolar limitan o facilitan esta construcción?

Por su parte Márquez (2014) planteó en su investigación el objetivo de conocer e interpretar cómo se llevan a cabo las prácticas evaluativas que realizan los docentes, así como sus sentidos en el sexto grado de educación primaria. Fue una investigación de tipo cualitativo y se realizó desde la perspectiva interpretativa. Sus conclusiones denotan que el examen es considerado como sinónimo de evaluación. Por su estructura, diseño y elaboración de dicho instrumento no se considera como el más pertinente para evaluar las competencias del alumno. La (RIEB), demanda una manera diferente de evaluar, por lo que es necesario transitar a un proceso en donde la evaluación se realice en un ambiente de confianza y de libertad para que reconozca sus aciertos y errores.

En la lógica de enunciar los antecedentes respecto a las prácticas de evaluación en el aula, en 2015 Martínez Rizo publica un artículo con el título "Estudios sobre prácticas de evaluación en el aula: revisión de la literatura" en donde magistralmente sintetiza los resultados de algunos de los estudios que han enfocado con más detalle las prácticas de evaluación en el aula, retoma en primer lugar al estudio de Stiggins y Conklin (1992) en el contexto Estadounidense, posteriormente retoma un estudio Latinoamericano de Laureiro, Ravela y Picaroni (2009) y finaliza con tres textos de investigaciones del contexto Mexicano.

En los trabajos de Stiggins y Conklin (1992) hacen referencia a estudios cuantitativos y cualitativos, uno de ellos se basa en una encuesta con 228 profesores de ocho regiones distintas de Estados Unidos en el que analizaron la práctica de 32 docentes mediante un diario que los mismos profesores realizaron, el análisis comprendió 290 actividades de evaluación el cual evidenció que el $75 \%$ de estas actividades tuvo el propósito de calificar si los alumnos dominaban los contenidos, el $18 \%$ fueron actividades de diagnóstico y el resto de retroalimentación.

Los estudios cualitativos describen la complejidad de las prácticas de evaluación que en estudios cuantitativos no se había podido apreciar. Además estos estudios llevados a cabo en educación media aportaron elementos como el nivel de demanda cognitiva de las evaluaciones, en este sentido, hubo prácticas que privilegiaban a la memorización de los contenidos, otras a la comparación de la información y muy pocos al análisis de textos. Se mostró en estos mismos estudios que para los docentes las pruebas estandarizadas tienden a ser confiables por lo que muchos de ellos las imitaban mientras que las evaluaciones con base 
a la observación del desempeño se usaron con menor frecuencia. Los docentes expusieron en estos estudios que ocupan aproximadamente el 25\% del tiempo en las actividades de evaluación que representa un menor porcentaje en relación al 40\% que dedica a la exposición de temas. Dichos estudios también revelan que la mayoría de los docentes consideran a la enseñanza y a la evaluación como procesos separados por lo que se dificulta encontrar una relación que los lleve a reflexionar sobre la naturaleza y la calidad de sus prácticas de evaluación.

Otro estudio de Stiggins y Conklin (1992) refiere a un proyecto latinoamericano que comprendió ocho países y se llevó a cabo en 80 escuelas con 160 profesores de sexto grado de primaria, mismos que respondieron un cuestionario y participaron en una entrevista semiestruturada, en su análisis ponen sobre la mesa que muchos docentes hacen una evaluación diagnóstica que toman como base para planificar sus actividades así como una evaluación al finalizar el bimestre. En esta investigación algunos docentes hacen referencia a la evaluación formativa y a la evaluación sumativa, en el discurso dicen preferir a la evaluación formativa pero en la práctica está ausente la retroalimentación y dan prioridad a la calificación, lo cual limita la comprensión de cómo habrán de mejorar.

En cuanto al estudio mexicano las prácticas de evaluación se enfocaron particularmente al contexto del estado de Nuevo León. Se trata de un análisis documental con base a entrevistas semi-estructuradas, mediante el cual se constató que el 68\% de los docentes aplica exámenes escritos cada bimestre, el $31 \%$ hace lo mismo pero de manera mensual, quincenal o semanalmente y tan sólo el 1\% no hace uso de exámenes. Martínez Rizo (2015) rescata en esta investigación que nueve de cada diez docentes dice informar a sus alumnos sobre los aprendizajes a evaluar en cada bimestre y los criterios de evaluación pero se contradice con la información que reportan los alumnos porque según ellos el $45 \%$ de los profesores explican en detalle cómo van a calificar mientras que el $34 \%$ lo hace de manera general y el resto que pocas veces lo hace o nunca. El 57\% de los alumnos refiere que la práctica más frecuente de su maestro es indicar los temas a estudiar en el examen, el 38\% expresa que el docente indica las páginas del libro para estudiar y el resto no ofrece ninguna información. Finalmente, en cuanto a la forma de devolución más común que hacen los docentes según esta investigación es de manera oral con el $37 \%$, con un $11 \%$ de los docentes que lo hacen por escrito y el resto únicamente hace saber la calificación sin ningún tipo de devolución. 
Como conclusión a la revisión que Martínez Rizo (2015) hace de estas investigaciones enfatiza que otras investigaciones sobre las prácticas de evaluación serán el mejor punto de partida de esfuerzos para que los profesores desarrollen su competencia para hacer evaluaciones que mejoren el aprendizaje de sus alumnos porque estas investigaciones han mostrado que muchas veces la práctica del docente no es congruente con sus concepciones y creencias.

\section{Construcciones epistemológicas de la evaluación. Recorrido histórico}

La evaluación en el aula encierra controversia y polémica desde distintas posturas ya sea ésta pedagógica, política, económica y social, de ahí la pluralidad de enfoques, estrategias, técnicas e instrumentos que delinean su desarrollo dentro del aula. La afirmación de que la evaluación tiene connotaciones ideológicas, sociales, pedagógicas y técnicas con expectativas e intereses particulares (Sanjurjo y Vera, 2003) tiene que ver con los fines de la educación y de la importancia que ésta guarda en la sociedad. En este sentido desde una visión pedagógica, "la evaluación en el aula es potencialmente una herramienta al servicio de la mejora de los aprendizajes y se la concibe integrada a la enseñanza" (Picaroni, 2009: 10), es así que la evaluación desde el contexto del aula, tiene implicación tanto con el proceso de enseñanza como con el proceso de aprendizaje pues se diseña en función del uso que se ha de otorgar a los resultados, la metodología y la orientación para favorecer el aprendizaje. Al posicionarse la evaluación dentro del contexto escolar, los actores educativos como docentes, directivos, padres de familia y alumnos le asignan una particular atención por ser un proceso que tiene implicación directa con la enseñanza y el aprendizaje.

En el mismo contexto escolar la evaluación como "disciplina científica" (Castillo y Cabrerizo, 2003) da apertura a una evolución del concepto no sólo en el discurso sino también en la práctica del docente y de los demás actores educativos. Es así que, la contextualización histórica de la evaluación parte de la reflexión de que es un concepto que viene del mundo de la industria con una profunda transformación desde que se implantó y se divulgó en el ámbito educativo, por lo que para tener una idea más clara cuando hablamos de evaluación me parece importante hacer un recorrido histórico a partir de los planteamientos de Stufflebeam y Shinkfield (1987) a través del cual se puede contextualizar el avance del concepto en el campo educativo para comprender su función social y provocar la innovación como producto de la investigación sobre la práctica docente. 
El análisis histórico de la investigación se basa en el trabajo de Ralph W. Tyler que es considerado el padre de la evaluación educativa, de ahí que se utilizan sus contribuciones iniciales como un punto de referencia central, para ello Stufflebeam y Shinkfield (1987) establecen cinco períodos básicos, los cuales enuncio enseguida:

\begin{tabular}{|l|l|}
\hline $\begin{array}{l}\text { Período de la evaluación } \\
\text { entendida como: }\end{array}$ & \\
\hline El período pre- Tyler & Abarca hasta 1930 \\
\hline La época tyleriana & Va desde 1930 hasta 1945 \\
\hline La época de la inocencia & Desde 1946 hasta 1957 \\
\hline La época del realismo & De 1958 a 1972 \\
\hline La época del profesionalismo & De 1973 hasta nuestros días \\
\hline
\end{tabular}

Tabla 1 Evolución del concepto de evaluación. Fuente: Stufflebeam y Shinkfield (1987). Elaboración propia.

En el período pre- Tyleriano la evaluación sistemática no era desconocida pero tampoco era un movimiento de mayor importancia. La larga historia de la evaluación data un poco más de 2000 años atrás con los Chinos, en el S. v a. C., Sócrates utilizó cuestionarios evaluativos como parte de su metodología didáctica. En 1845 en Estados Unidos, Horace Mann dirigía evaluaciones basadas en tests de rendimiento pero no se veían avances sustanciales en el aprendizaje. Gran parte de dichas evaluaciones eran destinadas a las Instituciones y programas de estudios. Desde el estudio que hacen Stufflebeam y Shinkfield una aproximación aún mayor a la evaluación se produjo en los primeros años del S. XX con la aparición de los tests estandarizados con la pretensión de que la educación fuera más eficiente.

A pesar de la larga historia de la evaluación muchas de las ideas y técnicas siguen presentes en la actualidad pues la fe ciega a las pruebas estandarizadas en México parece estar en boga en la política educativa, muchas veces sin responder a las condiciones de los centros escolares.

\section{Época Tyleriana}

La época Tyleriana se caracteriza porque en ella se acuñó el término evaluación educacional, fue durante aproximadamente 15 años en el que se amplió la visión del currículo y la evaluación hasta la conformación de un método que se centraba en unos objetivos claramente fijados. Personajes como John Dewey contribuyeron a que la escuela se convirtiera en un sistema dinámico e innovado lo cual dio lugar a la educación progresista donde se dejó ver 
la filosofía del pragmatismo y empleó los instrumentos del conductismo. En esta época la evaluación se estableció como un mecanismo de medida, concepción vista desde la psicología conductista de Skinner y Watson. "El paradigma conductista ha concentrado sus propuestas de aplicación en el llamado análisis conductual aplicado a la educación” (Hernández, 1998), el cual nombró a la conducta (los procesos observables) su objeto de estudio, lo que importaba era ver las diferencias individuales sin tomar en cuenta el proceso.

\section{Época de la inocencia}

La época de la inocencia o la época de la irresponsabilidad social como se le conoció según Stufflebeam y Shinkfield (1987) fue un tiempo para crear y desarrollar aptitudes, adquirir recursos y lograr disfrutar la vida que pobreza, prejuicios raciales y de segregación. En esta época la aportación de Cronbach fue muy importante para la evaluación educativa dado a que enfatizó que una calificación no era más útil como el análisis de por qué se llegó a ese resultado lo que después dio paso al diagnóstico de las necesidades y valoración de los avances de los niños menos favorecidos como finalidad principal de la evaluación.

\section{Época del realismo}

En la época del realismo según Stufflebeam y Shinkfield (1987), personajes como Scriven (1967), Stufflebeam $(1967,1971)$ y Stake (1967) propusieron nuevos modelos de evaluación que se alejaban de los hasta ahora propuestos. Estas nuevas conceptualizaciones reconocían la necesidad de evaluar las metas, examinar lo que se invertía, la prestación de servicios, así como determinar los resultados, fue así que a finales de los 60 y los primeros años de los 70 aumentó el debate de cómo debía ser concebida la evaluación.

En la época del profesionalismo (1973) el campo de la evaluación se cristalizó aún más y empezó a emerger como una profesión, aumentó la investigación sobre el tema pero no había literatura al respecto excepto trabajos inéditos que circulaban únicamente entre los especialistas y había muy pocas oportunidades de prepararse para ejercer un servicio evaluativo. Este periodo se caracterizó por la creciente búsqueda de métodos, técnicas y prácticas que se llevaron a cabo en cada periodo histórico (Stufflebeam y Shinkfield, 1987) pero al hacer un recuento de las prácticas de evaluación en la actualidad, se observa que la evaluación ha tenido pocos cambios pues aún destaca el uso de las pruebas estandarizadas así como otras estrategias evaluativas que no han sido sólo una moda como pudiera pensarse sino forman parte de la complejidad que envuelve a la evaluación. 
Como se ha contextualizado hasta ahora, la evaluación tiene un concepto multifacético desde distintas perspectivas y tendencias, en sus inicios en el campo de la educación como "sinónimo de medición de estados del alumno, de rendimientos de diverso tipo o de productos de aprendizaje" (Gimeno, 2010: 339) posteriormente, su práctica apuntó mirar las dificultades con la única consecuencia de mejorar el aprendizaje. Desde esta óptica parece una actividad sencilla o sin mayor complicación, sin embargo al pretender la objetividad de la evaluación se ha tecnificado de tal manera que se ve con normalidad muchas de sus prácticas con la finalidad de hacer de la pedagogía una práctica más científica, precisando sus objetivos y tecnificando los procedimientos de evaluación. Es así que, la cientificidad de los procedimientos visualizo que la evaluación se aleja de las condiciones particulares del contexto y de la individualidad de la persona al considerar la homogeneidad de instrumentos y las formas de emitir un juicio de valor estandarizado.

De manera que, analizar qué, cómo, cuándo y por qué evalúan los docentes en el aula así como la perspectiva desde dónde realizan su práctica se puede comprender en un sentido más amplio el significado que tiene la evaluación tanto para los docentes como para los alumnos. Desde mi punto de vista la evaluación es una práctica compleja por todo lo que converge en el aula como son contextos diversos, experiencias, modos de pensar, saberes y necesidades particulares. De ahí que no hay una práctica docente igual a otra porque no hay grupos idénticos y por lo tanto necesidades y condiciones diferentes, de este modo la evaluación en el aula resulta una tarea compleja porque "puede comprender prácticas muy diversas, concretarse en estilos muy diferentes, de acuerdo con las opciones que se adopten en cada uno de los pasos que hay que dar en ese proceso" (Gimeno, 2010: 344) de manera que al Institucionalizarse a la par del currículo se puede comprender un modo de pensamiento, intereses, normas y cultura del Sistema Educativo.

Es así que en un aula de clases pueden verse distintas prácticas de evaluación sustentadas en modelos, métodos y paradigmas que subyacen, convergentes o divergentes entre la comunidad educativa debido a que vivimos "momentos de acelerados cambios sociales y educativos,...nos enfrentemos a esas demandas de cambio educativo movidos por la inercia de concepciones o creencias implícitas nunca articuladas en las que fuimos formados" (Pozo, 2006: 13), dichos cambios llevan implícitos formas de pensamiento y tradiciones institucionales que configuran un modo de ser y actuar. 
En este sentido, la práctica evaluativa se entiende como un "conjunto de situaciones, más o menos relacionadas entre sí, que se distribuyen a lo largo de una unidad temporal de carácter educativo." (Coll y Rochera, 2012: 54), en dichas situaciones puede observarse el diseño de un plan de evaluación que tenga la finalidad de apreciar el aprendizaje de los estudiantes mediante un tipo de evaluación formal e informal que le ofrezcan un espacio de reflexión, además de contar con una amalgama de elementos que configuren la articulación de la práctica.

\section{3. ¿A qué nos referimos cuando hablamos de prácticas del aula en la escuela primaria?}

En este apartado retomo los distintos significados que tienen las prácticas en el aula de la escuela primaria, todas ellas implican una red de interacciones, decisiones, experiencias e ideologías de los profesores lo cual da razón a todas las acciones que tienen lugar en el hacer docente. Coincido con la idea de que una persona hace cosas que conoce, que están en su repertorio de posibilidades o que en definitiva decide que es mejor (Sañudo, 2015) frente a una determinada situación. De modo que, la práctica toma sentido a partir del ámbito en el que se llevan a cabo dichas acciones pero ¿cómo saber qué determina una práctica?, ¿cuáles son sus partes?

La palabra práctica desde la semántica deriva el uso continuado, la costumbre o estilo de hacer algo, es desde este punto de vista un concepto que abona a todas las disciplinas pero que a su vez se distingue en cada una por la esencia que la constituye. Significar la práctica implica un rastreo del concepto pues vista desde la práctica científica toma un carácter experimentado, versado y diestro en algo, desde las artes puede comportar utilidad o produce provecho material inmediato, desde la milicia puede considerarse como el ejercicio de cualquier facultad conforme a sus reglas, la práctica religiosa por su parte se orienta hacia la aplicación de una idea o doctrina (Pérez, 2010) sin embargo, en esta investigación hago énfasis en las prácticas que tienen lugar en el ámbito educativo, particularmente a las prácticas de evaluación que se desarrollan en el aula.

En este sentido, la práctica tiene lugar en el ejercicio de una actividad que se puede acentuar como habilidad, experiencia o hábito, por lo que algunas veces la práctica puede denotar un conocimiento o algunas veces una necesidad según sea la esencia que la distingue. "En suma, 
la práctica se ha presentado como razón que funda nuestros saberes y como motivo que determina nuestros procesos de justificación" (Villoro, 1982, p. 251), por lo que una persona echa mano de las ideas a partir del conocimiento, la reflexión y la experiencia. De manera que la práctica señala indicios de que detrás de ella hay un conocimiento, tiene un sentido social y hay una verdad justificada en la creencia traducida en disposición a actuar de una u otra manera.

Como puede notarse las prácticas no son exclusivas de lo educativo por lo que enfatizo que en este trabajo el concepto de práctica se enfoca desde el contexto escolar donde los profesores desarrollan su trabajo docente. Así al observar las prácticas de evaluación de los profesores me llevó a reconocer los significados que dieron origen a las distintas actividades desarrolladas en el aula. En este sentido, para profundizar sobre dichas prácticas de evaluación como una de las tareas que el docente realiza fue necesario indagar sobre el sentido que se le otorga y sobre la cultura de evaluación que comparten los profesores de la investigación.

Hay quien afirma que, “....es en la práctica donde todo proyecto, toda idea, toda intención, se hace realidad de una forma $\mathrm{u}$ otra; se manifiesta, adquiere significación y valor [...] El currículum, al expresarse a través de una praxis, cobra definitivo significado para los alumnos y para los profesores" (Gimeno, 1991: 240), por lo que la actuación de los docentes responde al diseño y organización de contenidos previamente establecidos desde el Sistema Educativo. Entonces, la práctica responde no sólo a las exigencias curriculares sino también a las intenciones y posibilidades o alcances del profesor.

El docente dispone para su práctica teorías inconexas, desarticuladas, inestables, formada a lo largo de su experiencia como alumno y como profesor (Gimeno, 1991), elementos que son visibles en el aula de clases al momento de interactuar, establecer acuerdos o tomar decisiones. De manera que la práctica docente está intrínsecamente conectado con un conjunto de factores sociales y culturales vivenciados a lo largo de la formación ${ }^{4}$. De este modo, "la estructura de la práctica obedece a múltiples determinantes, tiene su justificación en parámetros institucionales, organizativos, tradiciones metodológicas, posibilidades reales

\footnotetext{
${ }^{4}$ Manera de ver integralmente el proceso que siguen los individuos para estar en y con su entorno -Bildung(Hegel, 1991).
} 
de los profesores [...] la práctica es algo fluido, huidizo, difícil de limitar con coordenadas simples" (Zabala, 2000: 14), por lo que estos múltiples factores hacen a la práctica docente una práctica compleja por los hábitos, valores, ideas y condiciones en el que se encuentran sumergidos.

Derivado de una práctica docente y de una práctica educativa se desprenden las prácticas de evaluación en el aula es decir; el proceso que manifiesta la manera en que el profesor revisa los trabajos, el tipo de retos y ayudas que se proponen, las manifestaciones de las expectativas depositadas, las notas que hace el profesor en el cuaderno de los alumnos, las valoraciones informales sobre el trabajo que se realiza de manera cotidiana, la manera de distribuir el grupo, las tareas que encarga el docente, son factores que ofrecen información sobre la concepción que se tiene de la evaluación. En este sentido al interrogar a los agentes participantes de la investigación denotan como un asunto obligado a la evaluación del proceso de enseñanza y aprendizaje, del mismo modo que si se pregunta qué y a quién evaluar se dejan ver tanto al objeto como al sujeto o sujetos de evaluación, por lo tanto, prestar atención en el cómo, con qué y cuándo evaluar representan marcos de referencia respecto a la función que tiene la evaluación en la práctica docente y práctica educativa presente en el aula.

\section{¿Para qué evaluar desde la perspectiva de los docentes?}

Cuando a los profesores involucrados en la investigación se les preguntó ¿para qué evaluar? fue evidente en su discurso que la mejora de la práctica docente es una prioridad para ellos lo cual traducen a que un mayor número de alumnos logren los objetivos planteados en términos de competencias con base a su entorno real. "La consecución de los objetivos por parte de cada alumno es un hito que exige conocer los resultados y los procesos de aprendizaje que los alumnos siguen. Y para mejorar la calidad de la enseñanza hay que conocer y poder valorar la intervención pedagógica del profesorado” Zabala, 2000: 209). De manera que el proceso de aprendizaje y el de enseñanza son objetos de evaluación que pueden informar sobre la actuación en el aula, por lo que se puede conceptualizar las prácticas de evaluación como una construcción sociocultural de los docentes a partir de la experiencia, la interacción con los otros, la formación y la cultura institucional.

Algunos estudios realizados en México revelan que los docentes dan gran importancia a sus propias observaciones al momento de evaluar, los docentes elaboran pruebas estandarizadas 
que aplican a todo el grupo pero expresan tener dificultad para elaborar instrumentos que demandan distintos tipos de contenido.

Considerar a la enseñanza y a la evaluación como dos procesos distintos o no encontrar la relación entre ellos en la práctica docente habla de un pensamiento funcionalista donde cada parte contribuye de un modo dogmático, dirigido y con un "supuesto" equilibrio. Conocer ciertos conceptos y desarrollarlos en la práctica hay gran distancia cuando en el discurso de muchos docentes se centra en las funciones de una evaluación sumativa y formativa, pero poco clara. Los docentes son capaces de caracterizar a la evaluación de manera general pero incurren en contradicciones, situación que permite suponer poca solidez de los conceptos expresados (Picaroni, 2009).

\section{Caracterización de las prácticas (trabajo empírico)}

En este apartado se enuncian algunos elementos que configuran las prácticas de evaluación en el aula los cuales fueron configurando las Unidades de Análisis ${ }^{5}$ que integran el trabajo de investigación. Gracias al empleo de algunas técnicas como notas de campo, entrevistas, historias de vida y conversaciones informales se identificaron recurrencias entre los sujetos, se elaboró un coloreograma en el que con base a distintos colores se configuraron las siguientes Unidades de Análisis:

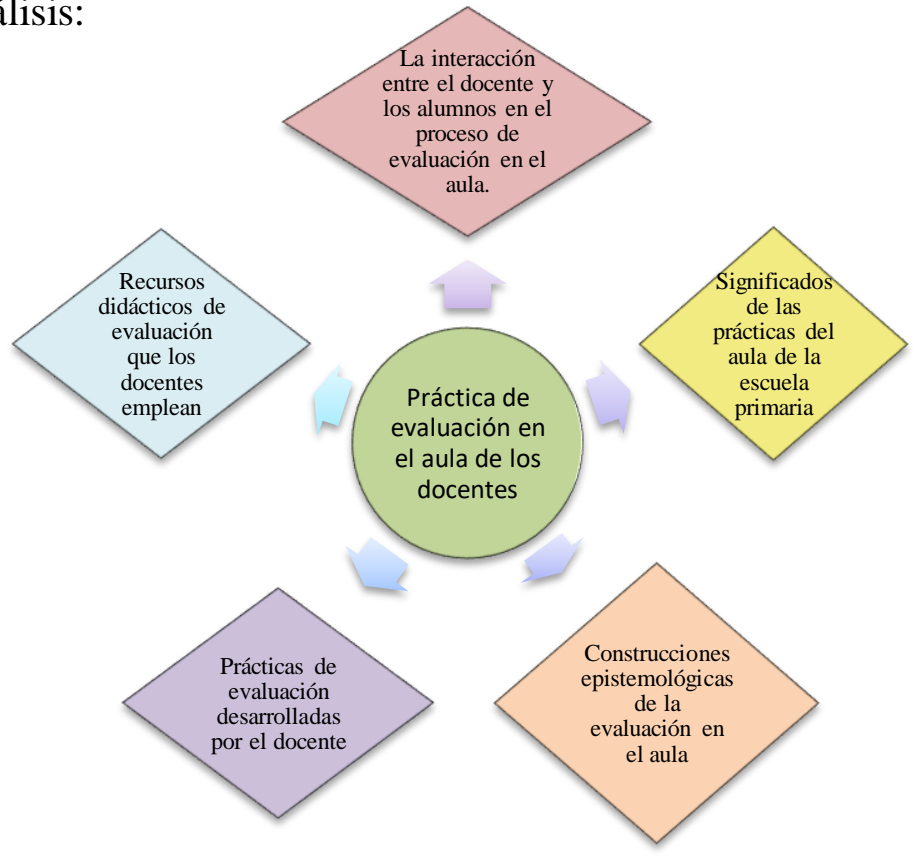

\footnotetext{
${ }^{5}$ Es el elemento (persona, Institución u objeto) del que se obtiene información fundamental. Pueden existir diversas unidades de análisis [...] dependiendo de los objetivos del estudio (Rojas, 2002, p. 180).
} 
Las unidades de análisis enunciadas surgen en el trabajo de campo en función de los objetivos planteados, por lo que en el texto se encuentran distintas nomenclaturas que remiten a la referencia de dónde se obtuvo la información como los registros de observación, entrevistas, historias de vida y conversaciones informales, por ejemplo: R1OEA/25-08-2017 ${ }^{6}$, EProfa. E/08-10-2017,$\quad$ HVProf.S/11-09-2017 ${ }^{8}, \quad$ CIProf $^{\mathrm{a}} . \mathrm{D} / 02-11-2017^{9}, \quad$ EAo.3/07-12-2017 ${ }^{10}$ mediante los cuales se pretende mejor comunicación del texto. De la misma manera se considerara el siguiente esquema para referirse a las escuelas y a los docentes:

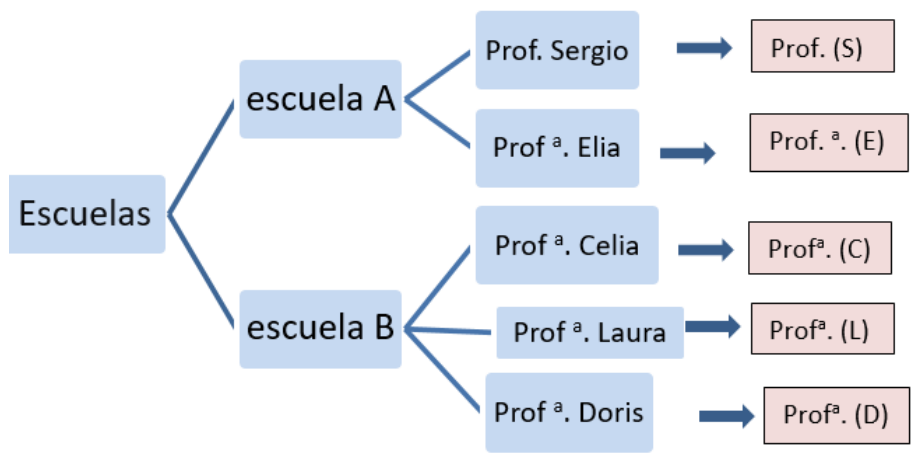

\section{La interacción del docente y los alumnos durante el proceso de evaluación en el aula}

Para describir y analizar las prácticas de evaluación que los profesores desarrollan en el aula se consideró conveniente contextualizar la dinámica de trabajo que se lleva a cabo en clase para identificar la relación que guardan las redes de interacción entre los sujetos que allí comparten, así como la comunicación, los referentes pedagógicos, condiciones del entorno escolar, connotaciones ideológicas, sociales, pedagógicas y técnicas empleadas con las prácticas de evaluación que allí se desarrollan por tratarse el aula de un "sistema vivo y complejo, comunicativo, en el cual los elementos se definen en función del intercambio" (Mallart, 2015: 38). De manera que los fenómenos que en ella se presentan adquieren un carácter simultáneo e inmediato por implicar a sujetos con vida propia y común del grupo, así las experiencias de evaluación que se gestan en el aula demandan de la participación del profesor, los alumnos, autoridades y padres de familia.

\footnotetext{
${ }^{6}$ Registro de observación número 1 en la escuela A del día veinticinco de agosto del año 2017

${ }^{7}$ Entrevista a la profesora Elia el día ocho de septiembre del año 2017

${ }^{8}$ Historia de vida del profesor Sergio elaborado el 11 de septiembre del año 2017

${ }^{9}$ Conversación informal con la profesora Doris el día dos de noviembre del año 2017

${ }^{10}$ Entrevista al alumno tres de lista el día siete de diciembre del año 2017.
} 
De este modo, una de las primeras experiencias de interacción que se da entre el docente y los alumnos en el aula es al momento de la revisión de los trabajos que los estudiantes elaboraron como tareas en casa, es característico en cada uno de los grupos observados que al inicio de las clases se dedica un tiempo específico a la revisión de ejercicios extraescolares. En este sentido, los profesores demandan que los estudiantes hagan saber si cumplieron o no con la tarea del día anterior, tanto los docentes de la escuela A, como los de la escuela B, solicitan las libretas o libros de la asignatura que se aborda y no de todas las tareas a la vez. La prof ${ }^{\text {a }}$. E, pide que entreguen la tarea de español en el horario que les toca trabajar español al mismo tiempo que de manera particular solicita a algunos alumnos que guarden silencio para que el resto del grupo escuche que además de la libreta también habrán de atender alguna otra indicación como se aprecia en el siguiente diálogo:

Prof $^{a}$. E: sacamos libreta de español, de acuerdo al horario nos toca español, por favor Joana guardamos silencio.

Aol: maestra ¿también sacamos el libro?

Prof ${ }^{a}$. E: Si, sacamos libro y libreta de español. Me traen la libreta, recuerden que antes de empezar revisamos la tarea. Por favor venga la fila uno, luego la dos y así para que no se amontonen en el escritorio (RIOEA/25-08-2017)

Se aprecia que por los pasillos de las filas de bancas varios alumnos caminan, algunos establecen breves conversaciones entre ellos y se detienen un poco para mostrar cómo hicieron la tarea, cuando la docente se percata de que algún alumno no se levanta para entregar la libreta o el trabajo se escuchan algunas frases como "Estoy esperando tu libreta Luis" (R1OEA/25-08-2017) o la docente camina hacia donde se encuentra sentado el alumno como para constatar de su cumplimiento.

La interacción entre el profesor y los alumnos se basa en preguntas y respuestas que llevan al alumno a saber que hacer durante las clases. En este sentido, la Prof ${ }^{\mathrm{a}}$. E es quien organiza el trabajo y suele decidir la asignatura que se va a trabajar, el tema que se va abordar, el tiempo que se va a ocupar, así como las actividades a realizar, el papel que muestra para con sus alumnos es de autoridad.

Los alumnos están habituados a entregar la tarea antes de iniciar la clase de la asignatura señalada, se observa que en pocos minutos la profesora devuelve las libretas con el apoyo de dos alumnos que se encargan de llevar la libreta correspondiente al asiento de sus compañeros, aunque no siempre son los mismo quienes entregan las libretas pues parece una actividad que llama la atención de los alumnos de quinto grado, se observa que algunos 
levantan la mano y pronuncian frases como "yo le ayudo maestra, yo entrego las libretas" (R1OEA/25-08-2017), en ocasiones la docente responde con frases como "Si, también tu Jimena, ayúdame por favor a repartir las libretas" (R1OEA/25-08-2017).

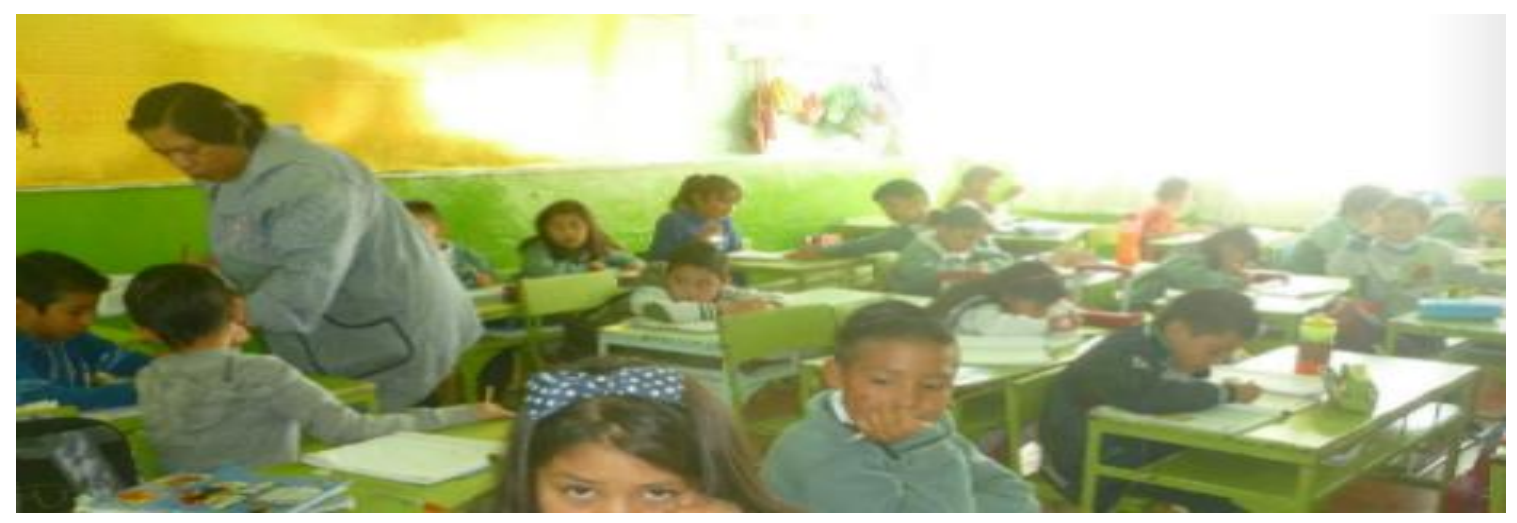

Interacción en el aula entre el profesor y los alumnos

Entender a la evaluación como el proceso integral que acompaña la enseñanza y el aprendizaje para poner en balance el avance o dificultades que tiene el alumno permitirá tomar decisiones de mejora a partir de la información obtenida. Hablar de evaluación nos conduce a mirar la manera en cómo se hace, qué se hace y para qué se hace cada actividad en el aula así como los rasgos normativos, formativos, cotidianos, la función que cumple y las razones en las que "se apoya en una serie de ideas y formas de realizarla" (Gimeno, 2010: 334) con base a las condiciones que caracterizan el contexto del aula y la cultura de cada docente. Registrar si el alumno cumplió o no con las tareas extraescolares es uno de los primeros momentos de evaluación en el aula que consideran los docentes cada uno en su estilo propician la interacción y un primer momento de evaluación al revisar lo que se demanda como tarea que se deriva de ello una escala estimativa elaborada en una tabla de doble entrada, instrumento que toma relevancia en el proceso de evaluación.

\section{Conclusiones}

Las reglas de interacción que guían al grupo es condicionado, impuesto y controlado por el docente pues las expectativas que se tienen de los estudiantes ya están decididas no sólo por el currículum sino por la organización y reglas anticipadas del profesor. En este sentido, se visualiza una pluralidad de enfoques, estrategias, técnicas e instrumentos que delinean los docentes en su práctica dentro del aula, si bien las tareas extraescolares tienen implicación con el aprendizaje de los alumnos, el mecanismo que se desarrolla en su revisión y que luego 
impacta en la evaluación se devalúa frente al proceso integral de la enseñanza porque la actividad adquiere un carácter de mero cumplimiento.

En este sentido, la interacción que se gesta en el aula ofrece información sobre la naturaleza,

el papel y la calidad no sólo de las estrategias de enseñanza sino también de las prácticas de evaluación, aunque hablar de interacción es apenas uno de los elementos que configuran el trabajo que se realiza en el aula, considero que aún hace falta reflexionar sobre el ser y hacer diario como punto de partida para tomar decisiones de mejora con los alumnos.

\section{Referencias}

Bertely, M. (2000) Conociendo nuestras escuelas. Un acercamiento etnográfico a la cultura escolar. México: Paidós

Bourdieu, P. (2002). Teoría del mundo social. Buenos Aires: Siglo XXI editores.

Duhalde, M. (1999). La investigación en la escuela. México: Ediciones novedades educativas.

Gimeno, J. y Pérez A. (2010) Comprender y transformar la enseñanza. Madrid: Morata.

Márquez, S., (2014). El proceso de evaluación en el sexto grado de educación primaria. (Tesis Doctoral). México: ISCEEM.

Martínez, R. F., (2011) La evaluación en el aula: promesas y desafíos de la evaluación formativa. México: Universidad Autónoma de Aguascalientes.

Martínez Rizo, F. (2015). La evaluación formativa del aprendizaje en el aula en la bibliografía en inglés y francés. Revisión de literatura. Revista Mexicana de Investigación Educativa, 17(54), 849-875.

Picaroni, B. (2009). La evaluación en las aulas de primaria: usos formativos, calificaciones y comunicación con los padres. Universidad Católica del Uruguay y Grupo de Trabajo sobre Estándares y Evaluación [GTEE-PREAL].

Pérez, G. (1994). Investigación cualitativa: retos e interrogantes. Madrid, España: Muralla. Rockwell, E. (1987) Reflexiones sobre el proceso etnográfico. Documento de Investigaciones y Estudios avanzados, IPN, México.

Santos, M. (2003). Una flecha en la Diana: la evaluación como aprendizaje. Madrid: España: Nárcea.

SEP (2011). Plan de estudios, Educación Básica Primaria, México: SEP.

Scriven, M. (1967).La metodología de evaluación. En R. Tyler, R. Gagne y M. Scriven, Perspectivas de la evaluación curricular (pp. 39-83). Chicago, IL: Rand McNally.

Stiggins, R. y Conklin, N. (1992). En manos del docente: prácticas de evaluación de evaluación en el aula. Albany, Prensa de la Universidad Estatal de Nueva York.

Velasco, M. G. (2006). "Las prácticas cotidianas y los saberes docentes sobre la evaluación de los aprendizajes en la escuela primaria. Un estudio de caso" (Tesis de maestría) Instituto Superior de Ciencias de la Educación del Estado de México. ISCEEM Tejupilco. México. Zabala, A. (2000). La práctica educativa. Cómo enseñar. Barcelona: España. Electrónica. 